\title{
HIGHER ORDER MATRIX SUSY TRANSFORMATIONS IN TWO-DIMENSIONAL QUANTUM MECHANICS
}

\author{
F.Cannata $^{1, a}$, M.V.Ioffe ${ }^{2, b}$, A.I.Neelov ${ }^{2,3, c}$, D.N.Nishnianidze ${ }^{2,4, d}$ \\ ${ }^{1}$ Dipartimento di Fisica and INFN, Via Irnerio 46, 40126 Bologna, Italy. \\ 2 Sankt-Petersburg State University, 198504 Sankt-Petersburg, Russia \\ ${ }^{3}$ Institute of Physics, University of Basel, CH-4056 Basel, Switzerland \\ ${ }^{4}$ Kutaisi Technical University, 4614 Kutaisi, Republic of Georgia
}

The iteration procedure of supersymmetric transformations for the twodimensional Schrödinger operator is implemented by means of the matrix form of factorization in terms of matrix $2 \times 2$ supercharges. Two different types of iterations are investigated in detail. The particular case of diagonal initial Hamiltonian is considered, and the existence of solutions is demonstrated. Explicit examples illustrate the construction.

\section{Introduction}

Supersymmetric Quantum Mechanics (SUSY QM) [1], [2] has become a well-known powerful tool for the investigation of problems of modern non-relativistic Quantum Mechanics. The main achievements of SUSY QM during the last two decades involved isospectral pairs of one-dimensional systems. In particular, the conventional SUSY QM (with supercharges of first order in derivatives) was generalized [3], [4] to Higher order SUSY QM (HSUSY QM) with supercharges $n$-th order polynomials in momentum. This generalization induces the deformation of SUSY QM algebra: the anticommutator of supercharges is now a polynomial in the Superhamiltonian. The class of pairs of isospectral systems which are connected (intertwined) by components of supercharge is thus enlarged [4]. Two classes of second order supercharges were found: reducible, which can be written as a chain of consecutive first order transformations, and irreducible second order transformations. In both cases the

\footnotetext{
${ }^{a}$ E-mail: cannata@bo.infn.it

${ }^{b}$ Corresponding author. Phone:+7(812)4284553; FAX: +7(812)4287240; E-mail: m.ioffe@pobox.spbu.ru

${ }^{c}$ E-mail: alexey.neelov@unibas.ch

${ }^{d}$ E-mail: qutaisi@hotmail.com
} 
intertwined Hamiltonians are expressed in terms of only one arbitrary function [4]. For a (admittedly nonexhaustive) list of references devoted to one-dimensional HSUSY QM see, in particular, [5].

It is also useful to recall the equivalent terminology related to the classic mathematical paper by G.Darboux [6]. The conventional first order SUSY transformations coincide with the so-called Darboux transformations, which are very useful in Mathematical Physics (see, for example, [7] for nonlinear evolution equations). Then $n$-th order HSUSY transformations correspond to the well-known Crum-Krein transformations [8], where the eigenfunctions of $n$-th iterated Hamiltonian are obtained in terms of $n$ given eigenfunctions of the initial Hamiltonian by means of "determinant expressions". The second iterations of SUSY transformations for matrix one-dimensional potentials were considered in [9], recently the general form of matrix Crum-Krein transformations was constructed [10].

Much less attention was paid in the literature to SUSY QM of two-dimensional systems. Here we list some partial achievements which have been obtained in this field. The direct multi-dimensional generalization of the standard one-dimensional SUSY QM was elaborated in [11], [12] for arbitrary space dimensions. In the simplest two-dimensional case the supersymmetrization of a given (scalar) Schrödinger Hamiltonian involves not only a second scalar Hamiltonian but also a matrix $2 \times 2$ Schrödinger operator. The spectra of these three components of the Superhamiltonian are interrelated, and their wave functions are connected by the components of supercharges. This approach was used successfully [13], [14] to find the spectrum and the wave functions of Pauli operators describing spin $1 / 2$ particles (with arbitrary values of the gyromagnetic ratio) in a broad class of nonhomogeneous external electromagnetic fields in terms of spectra and eigenfunctions of two scalar Hamiltonians.

In the series of papers [15] direct intertwining relations between a pair of scalar twodimensional Hamiltonians were studied. A wide variety of particular solutions was found, leading to new integrable quantum systems which are not amenable to separation of variables. This approach allowed to introduce [16] two new methods for the study of the twodimensional Schrödinger equation: SUSY-separation of variables and shape invariance. Multi-dimensional SUSY QM for an arbitrary oriented Riemannian manifolds was studied in [17]. In a very recent paper [18] the Hamilton-Jacobi approach of Classical Mechanics was applied to study classical two-dimensional supersymmetric models and their quantization. 
The present paper addresses the problem of the construction of a chain of consecutive supersymmetric (generalized Darboux) transformations for two-dimensional Schrödinger systems allowing for a deeper insight into isospectrality in multidimensional cases. Let us remark that the only early attempt to construct such iterations failed (see Section 4 of the first paper in [11]). Even the very possibility of making iterations for two-dimensional systems remained a task to be solved.

The new matrix supersymmetric factorization of the two-dimensional Pauli operator, recently proposed in [14], can provide a new chance to construct iterations of first order (reducible) supersymmetric transformations. Indeed, in contrast to the method of quasifactorization [11], where the vector components of supercharges include scalar superpotential, the matrix factorization of [14] involves the components of supercharges with matrix superpotentials. Therefore all elements of the SUSY intertwining relations are now represented by $2 \times 2$ matrix differential operators.

The organization of the paper is the following. In Section 2 the chain of of SUSY transformations is performed by gluing the corresponding components of two successive Superhamiltonians. The generalization of this prescription includes an additional global unitary transformation (Section 3). In Section 4 we analyze the particularly interesting case when the initial Hamiltonian is diagonal, and one supersymmetrizes a scalar Hamiltonian by building partner Hamiltonians via second order SUSY transformations. This construction, in reverse order, can be also used for diagonalization of a class of two-dimensional $2 \times 2$ matrix Schrödinger operators or for matrix systems of coupled second order differential equations.

\section{Second iteration by gluing}

Before introducing the new form of SUSY factorization for two-dimensional systems, proposed in [14], let us start from the main formulas for the conventional [2] one-dimensional SUSY QM with partner Hamiltonians and their eigenfunctions:

$$
\begin{array}{ll}
H^{(0)}=q^{+} q^{-}=-\partial^{2}+V^{(0)}(x) ; & H^{(0)} \Psi_{n}^{(0)}(x)=E_{n} \Psi_{n}^{(0)}(x) ; \\
H^{(1)}=q^{-} q^{+}=-\partial^{2}+V^{(1)}(x) ; & H^{(1)} \Psi_{n}^{(1)}(x)=E_{n} \Psi_{n}^{(1)}(x) .
\end{array}
$$

The operators $q^{ \pm}$are defined as:

$$
q^{+}=-\partial+W(x) ; \quad q^{-}=\left(q^{+}\right)^{\dagger}=+\partial+W(x) ; \quad \partial \equiv d / d x,
$$


and they intertwine the components (1), (2) of the Superhamiltonian:

$$
H^{(0)} q^{+}=q^{+} H^{(1)} ; \quad q^{-} H^{(0)}=H^{(1)} q^{-}
$$

Then, up to normalization factors,

$$
\Psi_{n}^{(1)}(x)=q^{-} \Psi_{n}^{(0)}(x) ; \quad \Psi_{n}^{(0)}(x)=q^{+} \Psi_{n}^{(1)}(x)
$$

The intertwinings (3) are the most important relations of the SUSY QM algebra:

$$
\left\{\hat{Q}^{+}, \hat{Q}^{-}\right\}=\hat{H} ; \quad\left(\hat{Q}^{+}\right)^{2}=\left(\hat{Q}^{-}\right)^{2}=0 ; \quad\left[\hat{H}, \hat{Q}^{ \pm}\right]=0
$$

where the Superhamiltonian $\hat{H}$ and supercharges $\hat{Q}^{ \pm}$are:

$$
\hat{H}=\left(\begin{array}{cc}
H^{(0)} & 0 \\
0 & H^{(1)}
\end{array}\right) ; \quad \hat{Q}^{+}=\left(\hat{Q}^{-}\right)^{\dagger}=\left(\begin{array}{cc}
0 & 0 \\
q^{-} & 0
\end{array}\right) .
$$

This procedure of supersymmetric (Darboux) transformations from $H^{(0)}, \Psi_{n}^{(0)}(x), E_{n}$ to $H^{(1)}, \Psi_{n}^{(1)}(x), E_{n}$ was iterated [4] by reducible supercharges of second order in derivatives. This iteration leads to a polynomial deformation of SUSY algebra.

A two-dimensional generalization of standard SUSY QM [11] organized (in the Superhamiltonian) one matrix $2 \times 2$ and two scalar Hamiltonians ${ }^{e}$. Each scalar Hamiltonian is separately intertwined with the matrix Hamiltonian, but the spectra of the two scalar Hamiltonians are not related. This construction was based on the quasi-factorization of all three components of the Superhamiltonian in terms of the first order differential operators $q_{l}^{ \pm}=\mp \partial_{l}+\left(\partial_{l} \chi(x)\right)$ and $^{f} p_{l}^{ \pm}=\epsilon_{l k} q_{k}^{\mp} ; l, k=1,2$. A similar quasi-factorization with new supercharges, used in [11] to build an iteration of SUSY transformations, turned out to be unsuccessful since several necessary constraints were too complicated to be implemented [11]. In addition, it led to a growth of the matrix dimensionality of the new Hamiltonians.

In the present paper we use the method [14] based on the matrix factorization of components of the two-dimensional Hermitean Superhamiltonian ${ }^{g}$ :

$$
H^{(0)}=q^{+} q^{-}=-\Delta^{(2)}+\left(W_{i}\right)^{2}+\sigma_{1}\left(\partial_{2} W_{1}-\partial_{1} W_{2}\right)-\sigma_{3}\left(\partial_{1} W_{1}+\partial_{2} W_{2}\right)
$$

${ }^{e}$ For the case of an arbitrary dimensionality $d$ of coordinate space the Superhamiltonian contains $(d+1)$ components $H^{(n)}, n=0,1, \ldots, d$ with matrix dimensionality $C_{d}^{n}$, i.e. dimensionality of the space with definite fermionic occupation number $n$ (see details in [12]).

${ }^{f}$ The summation over repeated indices $(i=1,2)$ is implied here and below.

${ }^{g}$ Slightly different notations are chosen here in comparison with [14]. 


$$
\begin{aligned}
H^{(1)}= & q^{-} q^{+}=-\Delta^{(2)}+\left(W_{i}\right)^{2}+\sigma_{1}\left(\partial_{2} W_{1}+\partial_{1} W_{2}\right)+\sigma_{3}\left(\partial_{1} W_{1}-\partial_{2} W_{2}\right) \\
& \partial_{i} \equiv \frac{\partial}{\partial x_{i}} \quad(i=1,2) \quad \Delta \equiv \partial_{1}^{2}+\partial_{2}^{2}
\end{aligned}
$$

The $2 \times 2$ components $q^{ \pm}$of $4 \times 4$ supercharge $\hat{Q}^{ \pm}$(see Eq.(6)) are matrix differential operators of first order in derivatives ${ }^{h}$ :

$$
q^{ \pm}=\mp \partial_{1}-i \sigma_{2} \partial_{2}+\sigma_{1} W_{2}+\sigma_{3} W_{1} \quad q^{+}=\left(q^{-}\right)^{\dagger}
$$

where $W_{1,2}$ are real, and $\sigma_{i}(i=1,2,3)$ are standard Pauli matrices. By construction, these operators intertwine the Hamiltonians (7), (8) according to the standard relations (3) of SUSY algebra. The relations (4) - (6) of one-dimensional SUSY QM are satisfied as well, with the proviso that we deal now with $2 \times 2$ matrix differential operators $q^{ \pm}, H^{(0)}, H^{(1)}$ and two-component wave functions $\Psi_{n}^{(0)}, \Psi_{n}^{(1)}$. Isospectrality of the (matrix) Hamiltonians $H^{(0)}, H^{(1)}$ holds, as usual, except for the possible zero modes of $q^{ \pm}$.

To iterate supersymmetric transformations we impose the ladder equation (see [3], [4]) thus gluing the lower component $H^{(1)}$ of the Superhamiltonian $\hat{H}$ with the upper component $\widetilde{H}^{(0)}$ of the next Superhamiltonian $\hat{\widetilde{H}}$, defined by (7), (8) with $W$ replaced by $\widetilde{W}$ :

$$
H^{(1)}=\widetilde{H}^{(0)}+C, \quad C=\operatorname{const}(\text { real }) .
$$

Then the second order supercharge $Q^{+} \equiv q^{+} \tilde{q}^{+}$gives the following intertwining relations:

$$
H^{(0)} Q^{+}=Q^{+}\left(\widetilde{H}^{(1)}+C\right) \text {. }
$$

The conditions (10) can be rewritten as a nonlinear system of differential equations:

$$
\begin{aligned}
W_{i}^{2} & =\widetilde{W}_{i}^{2}+C ; \\
\partial_{2} W_{1} & +\partial_{1} W_{2}=\partial_{2} \widetilde{W}_{1}-\partial_{1} \widetilde{W}_{2} ; \\
\partial_{1} W_{1}-\partial_{2} W_{2} & =-\partial_{1} \widetilde{W}_{1}-\partial_{2} \widetilde{W}_{2} .
\end{aligned}
$$

It may be convenient to express these ladder equations in terms of complex function of two mutually conjugated complex variables

$$
W\left(z, z^{\star}\right) \equiv W_{1}\left(z, z^{\star}\right)+i W_{2}\left(z, z^{\star}\right) ; \quad z \equiv x_{1}+i x_{2} \quad z^{\star} \equiv x_{1}-i x_{2},
$$

\footnotetext{
${ }^{h}$ An analogous form of matrix supercharges was used also in [19] in the context of ParaSUSY QM.
} 
as follows:

$$
\begin{aligned}
\left|W\left(z, z^{\star}\right)\right|^{2} & =\left|\widetilde{W}\left(z, z^{\star}\right)\right|^{2}+C \\
\bar{\partial} W\left(z, z^{\star}\right) & =-\partial \widetilde{W}\left(z, z^{\star}\right) ; \\
\partial \equiv \frac{\partial}{\partial z} & =\frac{1}{2}\left(\partial_{1}-i \partial_{2}\right) ; \quad \bar{\partial} \equiv \frac{\partial}{\partial z^{\star}}=\frac{1}{2}\left(\partial_{1}+i \partial_{2}\right) .
\end{aligned}
$$

The general solution of Eq.(16) can be written by means of an arbitrary complex function:

$$
W\left(z, z^{\star}\right)=-\partial F\left(z, z^{\star}\right) ; \quad \widetilde{W}\left(z, z^{\star}\right)=\bar{\partial} F\left(z, z^{\star}\right) ; \quad F\left(z, z^{\star}\right) \equiv F_{1}\left(z, z^{\star}\right)+i F_{2}\left(z, z^{\star}\right)
$$

with real $F_{1}$ and $F_{2}$.

In terms of $F_{1}\left(z, z^{\star}\right), F_{2}\left(z, z^{\star}\right)$ Eq.(15) reads:

$$
\left(\partial_{1} F_{1}\right)\left(\partial_{2} F_{2}\right)-\left(\partial_{2} F_{1}\right)\left(\partial_{1} F_{2}\right)=C
$$

Its general solution is a general solution of the homogeneous equation summed to a particular solution of the inhomogeneous one. One can check that the general solution of the homogeneous equation requires that $F_{2}\left(x_{1}, x_{2}\right)$ depends on its $\operatorname{arguments}$ via $F_{1}\left(x_{1}, x_{2}\right)$ only:

$$
F_{2}\left(x_{1}, x_{2}\right)=\Phi\left[F_{1}\left(x_{1}, x_{2}\right)\right] ; \quad F\left(z, z^{\star}\right)=F_{1}\left(x_{1}, x_{2}\right)+i F_{2}\left(x_{1}, x_{2}\right)
$$

(and, of course, vice versa). In order to find the particular solution of inhomogeneous equation (18) one has to introduce new variables $y_{1}, y_{2}$ such that $y_{2} \equiv x_{2}$ and $y_{1}$ coincides with one of the solutions (19), e.g. $y_{1} \equiv F_{1}\left(x_{1}, x_{2}\right)$. In new variables Eq.(18) becomes:

$$
\frac{\partial F_{1}\left(x_{1}, x_{2}\right)}{\partial x_{1}} \frac{\partial F_{2}\left(y_{1}, y_{2}\right)}{\partial y_{2}}=C
$$

and its solution for $F_{2}$ in terms of $F_{1}$ is:

$$
F_{2}\left(x_{1}, x_{2}\right)=C \int d y_{2}\left(\frac{\partial F_{1}\left(x_{1}, x_{2}\right)}{\partial x_{1}}\right)^{-1}
$$

where the partial derivative is taken with $x_{2}=$ const, and after integration over $y_{2}$ the initial variables $x_{1}, x_{2}$ are to be reinserted. So the general solution of (18) is the sum:

$$
F_{2}\left(x_{1}, x_{2}\right)=\Phi\left[F_{1}\left(x_{1}, x_{2}\right)\right]+C \int d y_{2}\left(\frac{\partial F_{1}\left(x_{1}, x_{2}\right)}{\partial x_{1}}\right)^{-1}
$$

Thus two matrix two-dimensional Hamiltonians (components of new Superhamiltonian) $H^{(0)}$ (see expression $\left.(7)\right)$ and $\left(\widetilde{H}^{(1)}+C\right)$, which can be written as:

$$
\widetilde{H}^{(1)}+C=H^{(0)}+2 \sigma_{1} \partial_{1} \partial_{2} F_{1}\left(x_{1}, x_{2}\right)-2 \sigma_{3} \partial_{1} \partial_{2} F_{2}\left(x_{1}, x_{2}\right)
$$


are intertwined by the differential matrix operator (component of new supercharges):

$$
\begin{aligned}
Q^{+} & \equiv q^{+} \tilde{q}^{+}=\partial_{1}^{2}-\partial_{2}^{2}+2 i \sigma_{2} \partial_{1} \partial_{2}+ \\
& +\frac{1}{4}\left[\left(\partial_{2} F_{2}\right)^{2}+\left(\partial_{2} F_{1}\right)^{2}-\left(\partial_{1} F_{2}\right)^{2}-\left(\partial_{1} F_{1}\right)^{2}\right]+ \\
& +\sigma_{1}\left[\left(\partial_{1} F_{1}\right) \partial_{2}-\left(\partial_{2} F_{1}\right) \partial_{1}-\frac{1}{2}\left(\partial_{1}^{2}+\partial_{2}^{2}\right) F_{2}\right]- \\
& -\frac{i}{2} \sigma_{2}\left[\left(\partial_{1} F_{1}\right)\left(\partial_{2} F_{1}\right)+\left(\partial_{1} F_{2}\right)\left(\partial_{2} F_{2}\right)\right]+ \\
& +\sigma_{3}\left[\left(\partial_{2} F_{2}\right) \partial_{1}-\left(\partial_{1} F_{2}\right) \partial_{2}+\frac{1}{2}\left(\partial_{1}^{2}+\partial_{2}^{2}\right) F_{1} \partial_{1}\right]
\end{aligned}
$$

The SUSY algebra has now a polynomial form:

$$
\left\{\hat{Q}^{+}, \hat{Q}^{-}\right\}=\hat{H}(\hat{H}-C) ; \quad\left(\hat{Q}^{+}\right)^{2}=\left(\hat{Q}^{-}\right)^{2}=0 ; \quad\left[\hat{H}, \hat{Q}^{ \pm}\right]=0 .
$$

This algebra differs essentially from the deformed SUSY algebra for a class of two-dimensional systems investigated in a series of papers [15] in so far as it does not incorporate any nontrivial symmetry operators (no central extension): thus we have no positive indications of integrability of the systems $H^{(0)}, \widetilde{H}^{(1)}$.

Higher order iterations. In order to perform the next iteration of supersymmetric (Darboux) transformations one has to glue $\widetilde{H}^{(1)}$ with the first component $\widetilde{\widetilde{H}}^{(0)}$ of a new Superhamiltonian. This procedure follows the previous one: superpotentials are expressed in terms of derivatives of the same arbitrary complex function $\widetilde{F}\left(z, z^{\star}\right)$ :

$$
\begin{aligned}
\widetilde{W} & =\widetilde{W}_{1}+i \widetilde{W}_{2}=+\bar{\partial} F\left(z, z^{\star}\right)=-\partial \widetilde{F} \\
\widetilde{W} & =\widetilde{W}_{1}+i \widetilde{\widetilde{W}}_{2}=+\bar{\partial} \widetilde{F}\left(z, z^{\star}\right) .
\end{aligned}
$$

The last equality in (21) implies that in terms of an arbitrary function $f\left(z, z^{\star}\right)$ :

$$
F\left(z, z^{\star}\right)=-\partial f\left(z, z^{\star}\right) ; \quad \widetilde{F}\left(z, z^{\star}\right)=+\bar{\partial} f\left(z, z^{\star}\right) .
$$

It is difficult to find the general solution of the nonlinear equations (15) and the analogous one for $\widetilde{W}, \widetilde{W}$, but it is possible to find particular solutions with certain simplifying ansatzes. For example, explicit solutions can be obtained for $C=\widetilde{C}=0$ and $f\left(z, z^{\star}\right) \equiv|\phi(z)|^{2}$.

\section{Iterations by gluing and global transformations}

We now modify the procedure (namely, Eq.(10)) of the previous Section by gluing the Hamiltonians $H^{(1)}$ and $\widetilde{H}^{(0)}$ up to a global unitary transfomation $U$. Such a procedure 
allows to preserve the main details of the scheme, but it might extend the class of intertwined Hamiltonians.

Without loss of generality, we may restrict ourselves ${ }^{i}$ to $U=i \sigma_{3}$ :

$$
H^{(1)}=U \widetilde{H}^{(0)} U^{-1}+C, \quad U=i \sigma_{3} .
$$

In this case (15), (16) must be replaced by:

$$
\begin{aligned}
\left|W\left(z, z^{\star}\right)\right|^{2} & =\left|\widetilde{W}\left(z, z^{\star}\right)\right|^{2}+C ; \\
\partial_{1}\left(\widetilde{W}_{2}-W_{2}\right) & =\partial_{2}\left(W_{1}+\widetilde{W}_{1}\right) ; \\
\partial_{1}\left(\widetilde{W}_{1}+W_{1}\right) & =\partial_{2}\left(W_{2}-\widetilde{W}_{2}\right) .
\end{aligned}
$$

The Eqs.(25), (26) can be solved explicitly: $W$ and $\widetilde{W}$ are interrelated by an arbitrary holomorphic function $G(z)$ :

$$
W\left(z, z^{\star}\right)+\widetilde{W}^{\star}\left(z, z^{\star}\right)=G(z) ; \quad G(z) \equiv G_{1}+i G_{2} ; \partial_{1} G_{1}=\partial_{2} G_{2} ; \quad \partial_{1} G_{2}=-\partial_{2} G_{1} .
$$

The remaining nonlinear equation (24) for $G$ can be written as:

$$
G_{1}^{2}+G_{2}^{2}-2 W_{2} G_{2}-2 W_{1} G_{1}+C=0 .
$$

It can be solved explicitly as a linear algebraic equation for superpotentials $W_{1}, W_{2}$ in terms of an arbitrary holomorphic function $G(z)$. For an arbitrary $G(z)$ one can choose also an arbitrary function $W_{2}\left(z, z^{\star}\right)$ and find the functions $W_{1}\left(z, z^{\star}\right), \widetilde{W}_{1,2}\left(z, z^{\star}\right)$ from Eqs. (28), (27). Thus one obtains a class of Hamiltonians (7) for which the second order matrix supersymmetric transformations can be performed. The resulting Hamiltonian

$$
\widetilde{H}^{(1)}+C=H^{(0)}+2 \sigma_{1} \cdot\left(\partial_{1} W_{2}-\partial_{2} W_{1}+\partial_{2} G_{1}\right)+2 \sigma_{3} \cdot \partial_{1} G_{1}
$$

is intertwined with the initial Hamiltonian $H^{(0)}$ by the operator

$$
Q^{+}=q^{+} \sigma_{3} \tilde{q}^{+}
$$

and is therefore isospectral (up to zero modes of $Q^{ \pm}$) to $H^{(0)}$.

Example with hidden symmetry. Among many possible examples we consider the simple peculiar case when the resulting $\widetilde{H}^{(1)}$ coincides with the initial $H^{(0)}$ up to a constant:

$$
H^{(0)}=\widetilde{H}^{(1)}+C
$$

${ }^{i}$ From now on we will refer to such global unitary transformation as "rotation" even disregarding $i$. 
This can be achieved by imposing (see (29)) $G(z) \equiv i a z$ and

$$
W_{2}\left(z, z^{\star}\right)=\frac{1}{2}\left(a \rho+\frac{C}{\rho}\right) \cos \phi+\Theta(\rho) \sin \phi ; \quad W_{1}\left(z, z^{\star}\right)=\frac{1}{2}\left(a \rho+\frac{C}{\rho}\right) \sin \phi+\Theta(\rho) \cos \phi,
$$

where $\rho, \phi$ are polar coordinates, $a$ is a constant and $\Theta(\rho)$ is an arbitrary function. The Hamiltonian (30) then becomes:

$$
H^{(0)}=-\Delta^{(2)}+\frac{1}{4}\left(a \rho+\frac{C}{\rho}\right)^{2}+(\Theta(\rho))^{2}-a \sigma_{1}-\frac{1}{\rho}(\rho \Theta(\rho))^{\prime} \sigma_{3} .
$$

We stress that though the potential in (31) depends on $\rho$ only, its matrix structure in general prevents a standard separation of variables, typical for scalar problems. From the intertwining (now commutation) relations $\left[H^{(0)}, Q^{ \pm}\right]=0$ one can find two mutually commuting Hermitean symmetry operators:

$$
\begin{aligned}
& R^{-} \equiv \frac{1}{2 i}\left(Q^{+}-Q^{-}\right)=i a \partial_{\phi} \\
& R^{+} \equiv \frac{1}{2}\left(Q^{+}+Q^{-}\right)=-\sigma_{3} H^{(0)}+i a \sigma_{2} \rho \partial_{\rho}+\sigma_{3} \frac{1}{2} a \rho\left(a \rho+\frac{C}{\rho}\right)-\sigma_{1} a \rho \Theta(\rho),
\end{aligned}
$$

with column-eigenfunctions, common to $H^{(0)}, R^{ \pm}$:

$$
e^{i m \phi} \hat{\psi}_{m}(\rho)
$$

The first one, $R^{-}$, reflects the obvious symmetry of $H^{(0)}$ under rotations., but the second symmetry operator (33) realizes certain "hidden" symmetry and has the property:

$$
\left(R^{+}\right)^{2}+\left(R^{-}\right)^{2}=H^{(0)}\left(H^{(0)}-C\right) .
$$

One can notice that the spectrum of the Hamiltonian involves double $m \leftrightarrow-m$ degeneracy, and in addition a possible double degeneracy (for each value of $m$ ) associated with a columnfunction $\hat{\psi}_{m}(\rho)$. A pedagogical example illustrating the considerations above is obtained from (31) with $\Theta(\rho) \equiv 0$ and $C=0$ : it is exactly solvable after suitable rotation and leads to a pair of decoupled radial oscillators with frequiency $a$ and a relative ground state energy shift $2 a$.

Higher order iterations. In general, the procedure of gluing with a global rotation enlarges essentially the class of Hamiltonians for which our method is applicable. We now demonstrate this considering two options to construct third order transformations:

1. The first option reads:

$$
H^{(0)} \longrightarrow H^{(1)} \equiv \widetilde{H}^{(0)}+C \longrightarrow \widetilde{H}^{(1)}+C \equiv \sigma_{3} \widetilde{\widetilde{H}}^{(0)} \sigma_{3}+C+\widetilde{C} \longrightarrow \widetilde{\widetilde{H}}^{(1)}+C+\widetilde{C},
$$


where $\longrightarrow$ denotes the appropriate first order matrix SUSY transformation induced by $q^{+}, \tilde{q}^{+}, \tilde{\tilde{q}}^{+}$, and $\equiv$ represents the gluing. The first gluing is of the kind described in Section 2 (Eq. (10)), and the superpotentials $W\left(z, z^{\star}\right)$ and $\widetilde{W}\left(z, z^{\star}\right)$ satisfy Eqs.(17), (18). The second gluing is described in Eq.(23), and $\widetilde{W}\left(z, z^{\star}\right), \widetilde{\widetilde{W}}\left(z, z^{\star}\right)$ satisfy Eqs.(27), (28).

Eq.(28) can be rewritten as:

$$
\left(G(z) \partial F^{\star}+G^{\star}\left(z^{\star}\right) \bar{\partial} F\right)-|G|^{2}-\widetilde{C}=0,
$$

where $F\left(z, z^{\star}\right)$ still satisfies (18). We now restrict ourselves to the case $C=0$, for which Eq.(18) has trivial particular solutions with purely real or purely imaginary $F$. Then, e.g. for real $F$, Eq.(34) can be solved by replacing $z, z^{\star}$ by:

$$
t \equiv \int \frac{d z}{2 G(z)}+\int \frac{d z^{\star}}{2 G^{\star}\left(z^{\star}\right)} ; \quad \tau \equiv i\left(\int \frac{d z}{2 G(z)}-\int \frac{d z^{\star}}{2 G^{\star}\left(z^{\star}\right)}\right),
$$

since Eq.(34) in new variables reads:

$$
\partial_{t} F_{1}=|G|^{2}+\widetilde{C}
$$

A solution for $F_{1}$ :

$$
F_{1}=T(\tau)+\int|G|^{2} d t+\widetilde{C} t
$$

depends on two arbitrary functions - one holomorphic $(G(z))$ and one real $(T(\tau))$. The $F=F_{1}$ so obtained generates $W$ and $\widetilde{W}$ when inserted into (17) and (27) (in terms of the initial variables $\left.x_{1}, x_{2}\right)$. Thus one obtains a class of Hamiltonians $H^{(0)},\left(\widetilde{\widetilde{H}}^{(1)}+C+\widetilde{C}\right)$, which are intertwined (and are isospectral) by the third order operator $Q^{+}=q^{+} \tilde{q}^{+} \sigma_{3} \tilde{\tilde{q}}^{+}$.

2. The second option includes two gluings, both with $\sigma_{3}$-rotations:

$$
H^{(0)} \longrightarrow H^{(1)} \equiv \sigma_{3} \widetilde{H}^{(0)} \sigma_{3}+C \longrightarrow \widetilde{H}^{(1)}+C \equiv \sigma_{3} \widetilde{\widetilde{H}}^{(0)} \sigma_{3}+C+\widetilde{C} \longrightarrow \widetilde{\widetilde{H}}^{(1)}+C+\widetilde{C}
$$

The superpotentials $W\left(z, z^{\star}\right)$ and $\widetilde{W}\left(z, z^{\star}\right)$ has to be found from equations of the form (28), and then $\widetilde{W}\left(z, z^{\star}\right)$ and $\widetilde{W}\left(z, z^{\star}\right)$ from equations of the form (27). One can obtain the general solution of these equations purely algebraically in terms of two arbitrary holomorphic functions $G(z), \widetilde{G}(z)$ :

$$
W\left(z, z^{\star}\right)=\frac{G^{2} \widetilde{G}-|\widetilde{G}|^{2} G-\widetilde{C} G-C \widetilde{G}^{\star}}{\widetilde{G} G-\widetilde{G}^{\star} G^{\star}}
$$

$\widetilde{W}\left(z, z^{\star}\right), \widetilde{\widetilde{W}}\left(z, z^{\star}\right)$ being derived, again algebraically, from two equations of form (27). Thus one obtains a new class of Hamiltonians $H^{(0)},\left(\widetilde{\widetilde{H}}^{(1)}+C+\widetilde{C}\right)$, which are intertwined by the third order $Q^{+}=q^{+} \sigma_{3} \tilde{q}^{+} \sigma_{3} \tilde{\tilde{q}}^{+}$, and are therefore isospectral up to zero modes of $Q^{ \pm}$. 


\section{Second order supersymmetrization of scalar two- dimensional Hamiltonians.}

In this concluding Section we will discuss particular cases of Sections 2 and 3, when the initial Hamiltonian $H^{(0)}$ is constrained to be diagonal. The condition of diagonality of (7) reads:

$$
\partial_{2} W_{1}-\partial_{1} W_{2}=0 \quad \Leftrightarrow \quad W_{2}=\partial_{2} \chi\left(x_{1}, x_{2}\right) ; \quad W_{1}=\partial_{1} \chi\left(x_{1}, x_{2}\right),
$$

where $\chi\left(x_{1}, x_{2}\right)$ is an arbitrary real function. In terms of this function one has $H^{(0)}=$ $\operatorname{diag}\left(H_{11}^{(0)}, H_{22}^{(0)}\right)$ with

$$
H_{11}^{(0)}=-\Delta^{(2)}+\left(\partial_{i} \chi\right)^{2}-\left(\partial_{1}^{2}+\partial_{2}^{2}\right) \chi ; \quad H_{22}^{(0)}=H_{11}^{(0)}+2\left(\partial_{1}^{2}+\partial_{2}^{2}\right) \chi,
$$

which is intertwined (and therefore, isospectral, up to zero modes of supercharges (9)) with non-diagonal Hamiltonian $H^{(1)}$. The latter is obtained by inserting (37) into (8). From now on we study the iteration of SUSY transformations for the initial Hamiltonian (38), i.e. the compatibility of conditions (37) with the iteration algorithm.

Simple gluing. For the case $C=0$ in (10) the combination of Eq.(17) with Eq.(37) can be solved in terms of an arbitrary real function $\xi\left(x_{1}, x_{2}\right)$ :

$$
\begin{aligned}
F_{1}\left(x_{1}, x_{2}\right) & =\left(\partial_{2}^{2}-\partial_{1}^{2}\right) \xi\left(x_{1}, x_{2}\right) ; \\
F_{2}\left(x_{1}, x_{2}\right) & =-2 \partial_{1} \partial_{2} \xi\left(x_{1}, x_{2}\right) ; \\
\chi\left(x_{1}, x_{2}\right) & =\left(\partial_{1}^{2}+\partial_{2}^{2}\right) \xi\left(x_{1}, x_{2}\right) .
\end{aligned}
$$

The last condition to be taken into account is the interrelation (19) between $F_{1}$ and $F_{2}$ :

$$
\left(\partial_{2}^{2}-\partial_{1}^{2}\right) \xi\left(x_{1}, x_{2}\right)=f\left[\partial_{1} \partial_{2} \xi\left(x_{1}, x_{2}\right)\right] .
$$

Of course, one can not find the general solution of this nonlinear functional-differential equation, but one can find a variety of particular solutions. The simplest choice $f=$ const in (42) is trivial, since the corresponding Hamiltonian $H^{(1)}$ can be directly diagonalized by rotation. Some less trivial solutions of (39), (40), (42) can be found by requiring that the l.h.s. of Eqs.(39), (40) are e.g. $F_{i}=\lambda_{i} \xi^{k} ; \quad F_{i}=\exp \lambda_{i} \xi$, etc.

For the case $C \neq 0$ one can try similar techniques as used for the solution of Eq.(18) to solve now the equation (18) and (37) together with (17). The resulting equation reads:

$$
\left(\partial_{1}^{2}-\partial_{2}^{2}\right) F_{2}=2 \partial_{1} \partial_{2} F_{1} .
$$


Particular solutions of this equation can be found by suitable ansatzes like, for example:

$$
F_{1}\left(x_{1}, x_{2}\right) \equiv x_{1} g\left(x_{2}\right)+f_{2}\left(x_{2}\right) ; \quad \Phi\left(F_{1}\right) \equiv a F_{1}^{2}+b F_{1}+c+\frac{d}{F_{1}},
$$

where $g\left(x_{2}\right), f\left(x_{2}\right)$ can be determined by solvability conditions, the functional $\Phi\left(F_{1}\right)$ has been introduced in (20), and $a, b, c, d$ are constants. One can also enlarge the class of solutions by suitable nonsingular linear transformations of $x_{1}, x_{2}$.

Gluing with rotation. In this case the nonlinear equation (28) takes the form:

$$
\left(\partial_{1} \chi\right) G_{1}+\left(\partial_{2} \chi\right) G_{2}=1 / 2\left[G_{1}^{2}+G_{2}^{2}+C\right]
$$

or, in terms of variables $z, z^{\star}$ :

$$
\left(G(z) \partial+G^{\star}\left(z^{\star}\right) \bar{\partial}\right) \chi\left(z, z^{\star}\right)=|G(z)|^{2}+\frac{1}{4} C .
$$

It has the form similar to Eq.(34) of the previous Section except for the fact that $\chi\left(z, z^{\star}\right)$ is a real function. Therefore, the general solution of (43) is expressed in terms of $t, \tau$ of (35) via an arbitrary (real) function $\Lambda(\tau)$ :

$$
\chi\left(z, z^{\star}\right)=\Lambda(\tau)+\int|G(z)|^{2} d t+\frac{1}{4} C t .
$$

The initial diagonal Hamiltonian $H^{(0)}$ from (7) and (37) reads:

$$
\begin{aligned}
H^{(0)} & =-\Delta^{(2)}+4(\partial \chi)(\bar{\partial} \chi)-4(\partial \bar{\partial} \chi) \cdot \sigma_{3}= \\
& =-\Delta^{(2)}+|G|^{2}+\frac{1}{2} C+\frac{C^{2}}{16|G|^{2}}+\frac{\Lambda^{\prime 2}(\tau)}{|G|^{2}}+\frac{2 \Lambda^{\prime}(\tau)}{|G|^{2}} \int \partial_{\tau}|G|^{2} d t+\frac{1}{|G|^{2}}\left(\int \partial_{\tau}|G|^{2} d t\right)^{2}- \\
& -\sigma_{3} \cdot\left(G^{\prime}+G^{\star \prime}+\frac{\Lambda^{\prime \prime}}{|G|^{2}}\right)
\end{aligned}
$$

while the final $\widetilde{H}^{(1)}$ can be expressed as:

$$
\widetilde{H}^{(1)}+C=H^{(0)}-2 \sigma_{1} \partial_{1} G_{2}+2 \sigma_{3} \partial_{1} G_{1}
$$

As a particular example one can consider the case of $G=z^{1 / 2}$ which leads to a confining singular Hamiltonian with a singularity $1 / \rho$ and growing asymptotically as $\rho$ with a nontrivial azimuthal dependence.

Finally, we note that conversely the intertwining between diagonal $H^{(0)}$ and non-diagonal $\widetilde{H}^{(1)}$ by second order matrix supercharges can also be used to diagonalize a class of matrix Schrödinger operators (or system of differential equations of second order with non-diagonal matrix coupling), in analogy with similar procedures already used in [13], [14], [20]. 


\section{Acknowledgements}

M.V.I. and D.N.N. are grateful to the University of Bologna and INFN for support and kind hospitality. This work was partially supported by the Russian Foundation for Fundamental Research (Grant No.02-01-00499).

\section{References}

[1] E. Witten 1981 Nuclear Physics B185 513

[2] G. Junker 1996 Supersymmetric Methods in Quantum and Statistical Physics (Springer, Berlin)

F. Cooper, A. Khare, U. Sukhatme 1995 Phys. Rep. 25268

B.K. Bagchi 2001 Supersymmetry in Quantum and Classical Mechanics (Chapman and Hall/CRC, New York)

[3] A.A. Andrianov, M.V Ioffe, V.P. Spiridonov 1993 Phys.Lett. A174 273

[4] A.A. Andrianov, F. Cannata, J.-P. Dedonder, M.V. Ioffe 1995 Int.J.Mod.Phys. A10 2683

[5] B.F. Samsonov 1996 Mod. Phys. Lett. A11 1563

D.J. Fernandez C., M.L. Glasser, L.M. Nieto 1998 Phys.Lett. A240 15

V.G. Bagrov, B.F. Samsonov, L.A. Shekoyan 1998 quant-ph/9804032

S. Klishevich, M. Plyushchay 1999 Mod. Phys. Lett. A14 2739

A.A. Andrianov, F. Cannata, M.V. Ioffe, D.N.Nishnianidze 2000 Phys.Lett. A266 341

D.J. Fernandez C., J. Negro, L.M. Nieto 2000 Phys.Lett. A275 338

S. Klishevich, M. Plyushchay 2001 Nucl.Phys. B606[PM] 583

H. Aoyama, M. Sato, T. Tanaka 2001 Phys.Lett. B503 423

H. Aoyama, M. Sato, T. Tanaka 2001 Nucl.Phys. B619 105

R. Sasaki, K. Takasaki 2001 J. Phys. A: Math.Gen. 349533

A.A. Andrianov, A.V. Sokolov 2003 Nucl.Phys. B660 25

[6] G. Darboux 1882 Compt. Rend. 941456 
[7] F. Calogero, A. Degasperis Spectral Transform and Solitons v.1 (North-Holland Publishing Company, Amsterdam)

[8] M.M. Crum 1955 Quart. J. Math. Oxford 6121 (reproduced by H.Rosu in physics/9908019)

M.G. Krein 1957 Dokl. Akad. Nauk SSSR 113970 (in Russian)

[9] A.A. Andrianov, F. Cannata, M.V. Ioffe, D.N.Nishnianidze 1997 J. Phys. A: Math.Gen. 305037

[10] B.F. Samsonov, A.A. Pecheritsin 2003 quant-ph/0307145

[11] A.A. Andrianov, N.V. Borisov, M.V. Ioffe 1984 JETP Lett. 3993

A.A. Andrianov, N.V. Borisov, M.V. Ioffe 1984 Phys. Lett. A105 19

A.A. Andrianov, N.V. Borisov, M.V. Ioffe 1985 Theor. Math.Phys. 611078

[12] A.A. Andrianov, N.V. Borisov, M.V. Ioffe, M.I. Eides 1985 Phys. Lett. 109A 143

A.A. Andrianov, N.V. Borisov, M.V. Ioffe, M.I. Eides 1984 Theor. Math. Phys. 61965

[13] A.A. Andrianov, M.V. Ioffe 1988 Phys. Lett. B205 507

[14] M.V. Ioffe, A.I. Neelov 2003 J. Phys. A: Math.Gen. 362493

[15] A. Andrianov, M. Ioffe, D. Nishnianidze 1995 Phys.Lett., A201 103

A.A. Andrianov, M.V. Ioffe, D.N. Nishnianidze 1995 Theor. Math. Phys. 1041129

A.A. Andrianov, M.V. Ioffe, D.N. Nishnianidze 1996 solv-int/9605007; Published in: 1995 Zapiski Nauch. Seminarov POMI RAN ed.L.Faddeev et.al. 22468 (In Russian); Translation in: Problems in QFT and Statistical Physics ed.L.D.Faddeev et.al. 13

A.A. Andrianov, M.V. Ioffe, D.N. Nishnianidze 1999 J.Phys.:Math.Gen. A32 4641

[16] F. Cannata, M.V. Ioffe, D.N. Nishnianidze 2002 J.Phys.:Math.Gen. A35 1389

[17] A. Gonzalez-Lopez, N. Kamran 1998 J.Geom.Phys. 26202

[18] A. Alonso Izguierdo, M.A. Gonzalez Leon, M. de la Torre Mayado, J. Mateos Guilarte 2004 hep-th/0401054

[19] A. Andrianov, M. Ioffe, V.P. Spiridonov, L.Vinet 1991 Phys.Lett., B272 297 
[20] F. Cannata, M.V. Ioffe 1993 J.Phys.:Math.Gen. A26 L89 